\title{
Microgranular variant of acute promyelocytic leukemia with der(17) ins(17;15): A case report and review of the literature
}

\author{
HONGZAI GUAN ${ }^{1 *}$, JING LIU $^{2 *}$, XIAOFANG GUO ${ }^{1}$, CHUNMEI WU $^{1}$ and HUAWEI YU ${ }^{3}$ \\ ${ }^{1}$ Department of Clinical Hematology, Medical College of Qingdao University, Qingdao, Shandong 266071; \\ ${ }^{2}$ Clinical Laboratory of the Affiliated Hospital of Qingdao University, Qingdao, Shandong 266003; \\ ${ }^{3}$ Clinical Laboratory of No. 401 Hospital, PLA, Qingdao, Shandong 266071, P.R. China
}

Received August 14, 2014; Accepted May 7, 2015

DOI: $10.3892 / \mathrm{etm} .2015 .2583$

\begin{abstract}
Acute promyelocytic leukemia (APL) with variant translocations is rare. The patient of the present case report, a 2-year-old male with a microgranular variant of APL carrying der(17) ins(17;15) translocation, exhibited fever and epistaxis. The complete blood count showed marked leukocytosis with $72 \%$ atypical promyelocytes, anemia and thrombocytopenia. Conventional cytogenetic analysis of the bone marrow cells revealed a karyotype of 47, XY, add(3)(q29), -7 , ins(17;15)(q12;q14q22),+21,+mar. The promyelocytic leukemia/retinoic acid receptor $\alpha$ (PML/RAR $\alpha$ ) rearrangement and insertion were confirmed by fluorescence in situ hybridization. The PML/RAR $\alpha$ transcripts were not detected by the reverse transcription polymerase chain reaction, and the patient was diagnosed with microgranular variant M3 APL. The patient achieved remission after a 30-day treatment and was still in remission during a recent follow-up. The present findings suggest that the ins $(17 ; 15)$ variant in APL may not be associated with an unfavorable prognosis. In summary, we reported an extremely rare case of APL with der(17) ins $(17 ; 15)$ abnormality in a pediatric patient and reviewed the literature.
\end{abstract}

\section{Introduction}

Acute promyelocytic leukemia (APL) is a type of acute myeloid leukemia (AML) with distinct clinical, morphological, immunophenotypic and cytogenetic characteristics (1). APL has two morphological subtypes: Hypergranular (typical) APL

Correspondence to: Dr Huawei Yu, Clinical Laboratory of the No. 401 Hospital of the Chinese PLA, 22 Minjiang Road, Qingdao, Shandong 266071, P.R. China

E-mail: qdhongzaiguan@163.com

*Contributed equally

Key words: acute promyelocytic leukemia, microgranular acute promyelocytic leukemia, ins(17;15)(q12;q14q22), chromosomal aberrations, prognosis, fluorescence in situ hybridization and microgranular APL. Both subtypes are associated with disseminated intravascular coagulation. Unlike typical APL, the leukocyte count in microgranular APL is very high. The genetic hallmark of APL is $\mathrm{t}(15 ; 17)(\mathrm{q} 22 ; \mathrm{q} 21)$, which can be detected in $>90 \%$ of APL cases (2-5). The reciprocal translocation results in a fusion gene product between the retinoic acid receptor $\alpha(\mathrm{RAR} \alpha)$ gene on 17q21 and the promyelocytic leukemia (PML) gene on 15q22. This translocation is associated with particular sensitivity to treatment with all-trans retinoic acid (ATRA) (2) and a favorable prognosis; however, a subset of cases with APL morphology and variant translocations, such as $t(11 ; 17)$ and $t(5 ; 17)$, have been reported not to respond to ATRA (6) and have been associated with an unfavorable prognosis (7).

The present study describes a case of microgranular APL with ins(17;15)(q12;q14q22) in a pediatric patient, along with its clinical and biological characteristics. The case demonstrates the importance of taking into consideration all clinical, morphological and cytogenetic/molecular findings in the diagnosis of APL when typical morphological characteristics and/or cytogenetic findings are absent.

\section{Case report}

A 2-year-old boy, who had been suffering from recurring fever and cough for 5 days and epistaxis for 1 day, was admitted to the Affiliated Hospital of Qingdao University (Qingdao, China). He had an upper respiratory infection 5 days prior to admission. On admission, the patient was conscious but, according to physical examination, had pallor. No petechiae, xanthochromia or hemoptysis were observed and no hepatosplenomegaly or lymphadenopathy was noted during the examination. The head, chest and cardiovascular system examinations gave no notable results. The complete blood count differential showed that the white blood cell density was $4.628 \times 10^{10} / 1$ with $6 \%$ neutrophils and $72 \%$ atypical promyelocytes, the concentration of hemoglobin was $31 \mathrm{~g} / 1$ and the platelet density was $1.0 \times 10^{10} / 1$. Immunophenotyping by flow cytometry revealed that the atypical promyelocytes were positive for cluster of differentiation (CD) 13 (41.3\%) and CD33 (91.6\%) and negative for CD5, CD10, CD14, CD19, CD22, CD34 and human leukocyte antigen (HLA)-DR. Prothrombin time, partial thromboplastin time and the D-dimer test were within normal 
A

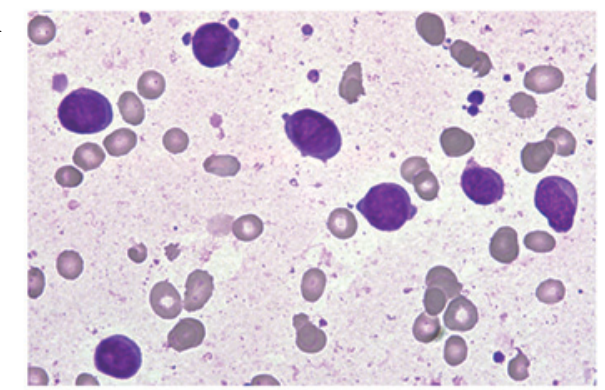

B

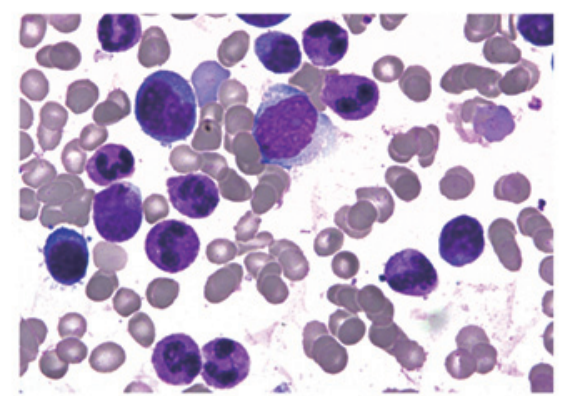

D

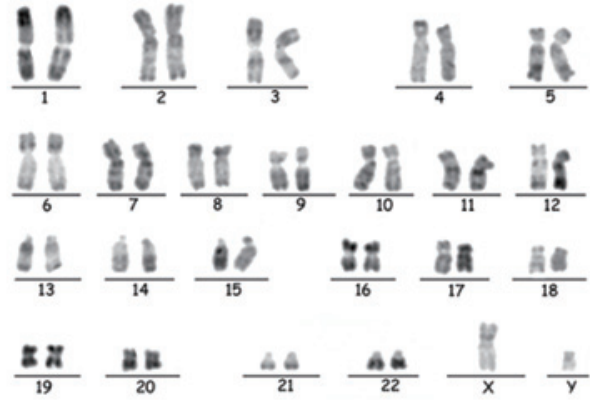

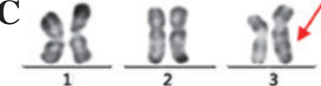

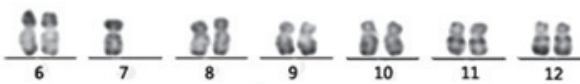
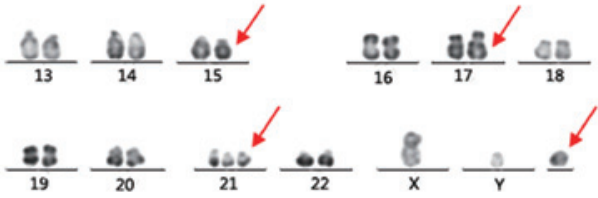

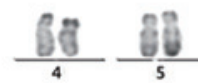

$\frac{19}{19} \frac{1}{20}$

$\mathbf{E}$

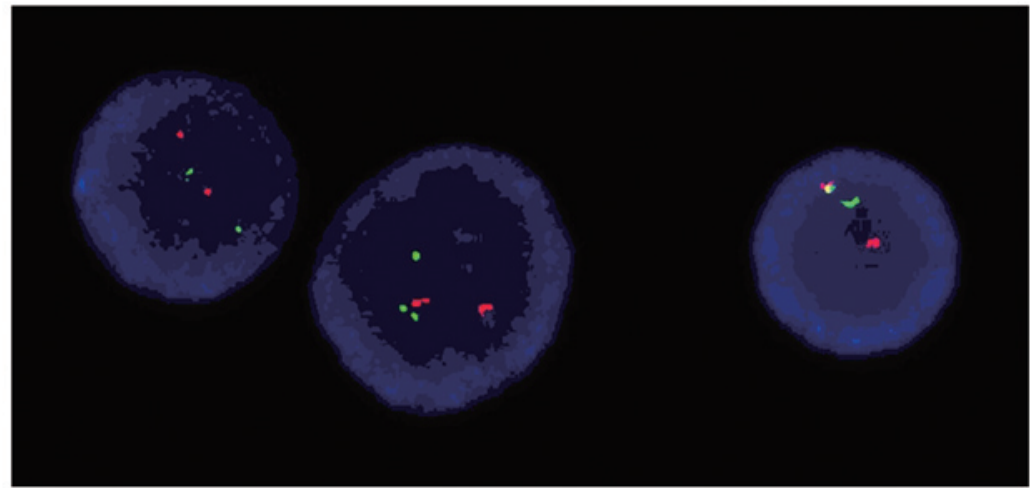

Figure 1. (A) Bone marrow aspiration smears showing hypoplastic and leukemic promyelocytes with round or oval nuclei, fine chromatin and the presence or absence of sparse small granules in the plasma at diagnosis (Wright stain; magnification, x100). (B) Bone marrow aspiration smears showing hyperplastic and rare promyelocytes with normal morphological characteristics when achieving complete remission (Wright stain; magnification, x100). (C) R-banded karyotype of a representative bone marrow cell: 47, XY, add(3) (q29), -7, ins(17;15)(q12;q14q22),+21,+mar. Arrows indicate derivative chromosomes. (D) R-banded karyotype of a representative bone marrow cell at clinical remission: 46, XY. (E) Fluorescence in situ hybridization of representative interphase cells with a PML/RAR $\alpha$ dual-color, dual-fusion translocation probe at diagnosis. Red signals represent the PML gene, green signals represent the RAR $\alpha$ gene and yellow signals represent the PML/RAR $\alpha$ fusion gene. PML/RAR $\alpha$, promyelocytic leukaemia/retinoic acid receptor $\alpha$.

ranges. Bone marrow aspiration was subsequently performed and the smears showed hypocellularity with $33.5 \%$ atypical promyelocytes. The majority of the leukemic cells were oval-shaped and slightly larger than their normal counterparts, whereas the occasional cells were irregular in shape, with abundant pauci-granular cytoplasm, round or oval nuclei and 1-4 nucleoli (Fig. 1A). In addition, there was an absence of Auer bodies. Bone marrow aspiration performed at complete remission showed $<5 \%$ immature cells (Fig. 1B). Prior written informed consent was obtained from the guardians of the patient and the study was approved by the Ethics Review Board of Qingdao University.

Chromosomal analysis. Chromosomal analysis was carried out following the standard protocol. In brief, the bone marrow aspiration samples were cultured in medium for $24 \mathrm{~h}$ and R-banding analysis was then performed. All 10 cells that were examined revealed a karyotype of $47, \mathrm{XY}$, add(3) (q29), -7, ins(17;15)(q12;q14q22),+21,+mar (Fig. 1C). A repeat karyotype analysis at clinical remission showed a normal karyotype (Fig. 1D).

Reverse transcription-quantitative polymerase chain reaction (RT-qPCR). RT-qPCR was performed to detect the transcripts of the PML/RAR $\alpha$ fusion gene. Total RNA was extracted using TRIzol reagent (Thermo Fisher Scientific, Waltham, MA, USA) from bone marrow aspirates, and complementary DNA (cDNA) was synthesized using total RNA (1-2 $\mu \mathrm{g})$ in a 40- $\mu$ l final reaction system containing reverse transcriptase M-MLV (Thermo Fisher Scientific) and random hexamers. The PCR primers were designed as previously described (8) The final volume of PCR was $50 \mu 1$, including cDNA $(3 \mu 1)$, primers (10 pmol each), dNTPs $(200 \mu \mathrm{M}), \mathrm{MgCl}_{2}(1.5 \mathrm{mM})$ and Taq DNA polymerase (0.3 U). An uninvolved RAR $\alpha$ gene segment was co-amplified as an inter-control. The products were detected on $2 \%$ agarose gels stained with ethidium 
bromide, transferred to positively charged nylon membranes (GeneScreen Plus ${ }^{\circledR}$; Perkin-Elmer Life Sciences, Waltham, MA, USA) and then hybridized using the probe R4b (5'-CTC ACAGGCGCTGACCCCCAT-3'), as previously reported (9). No PML/RAR $\alpha$ transcript was detected

Fluorescence in situ hybridization (FISH). FISH was performed on bone marrow interphases with the PML/RAR $\alpha$ dual-color DNA probe (obtained from Suzhou University, Suzhou, China), in which the PML and RAR $\alpha$ genes were labeled red and green, respectively. The detection was executed according to the manufacturer's instructions. Three signals (one red, one green and one yellow) were observed, indicating the presence of the PML-RAR $\alpha$ gene rearrangement (Fig. 1E). This observation may support the insertion observed in the conventional cytogenetic analysis.

Management and follow-up. The patient was initially treated with a supportive approach, and concentrated red blood cells and platelets were administered when necessary. Once the diagnosis of microgranular variant M3 (M3v) APL was rendered on the basis of the clinical and cytogenetic findings, the patient was treated with $10 \mathrm{mg}$ pirarubicin for 2 days, $30 \mathrm{mg}$ cytarabine for 4 days, $2 \mathrm{mg}$ arsenic trioxide for 5 days and $5 \mathrm{mg}$ ATRA twice a day. Four weeks later, the patient achieved complete remission and continued with consolidation therapy using pirarubicin and cytarabine.

\section{Discussion}

Hypergranular and microgranular APLs are both associated with high risks of disseminated intravascular coagulation and hemorrhagic manifestations, such as hematuria and epistaxis. According to the literature, microgranular APL accounts for $15-20 \%$ of APL cases $(6,10,11)$. Unlike hypergranular APL, leukemic cells in microgranular APL are pauci-granular or contain small, sparse cytoplasmic granules that sometimes can be mistakenly classified as monocytes $(12,13)$. As a result, $\mathrm{M} 3 \mathrm{v}$ is difficult to diagnose only by morphology and is often misdiagnosed as AML-M4 or -M5 (6). Cytogenetics and FISH studies are therefore indispensable for the correct diagnosis of $\mathrm{M} 3 \mathrm{v}$. In rare cases where typical morphological and clinical characteristics of APL are present or negative PML-RAR $\alpha$ rearrangement is observed in karyotyping, RT-PCR and FISH become necessary for the identification of rare variant translocations.

Regardless of the morphological variances, it appears that M3 and M3v have no significant differences regarding cytogenetic characteristics and clinical outcomes $(12,14)$. The PML/RAR $\alpha$ fusion product plays an important role in the pathogenesis of the disease by interfering with a normal cellular response towards retinoic acid. The classical translocation and presence of PML/RAR $\alpha$ rearrangement are generally associated with a favorable prognosis $(2,15)$; however, the presence of variant translocations and/or complex karyotypes may be associated with uncertain outcomes and prognosis $(16,17)$. For example, $\mathrm{t}(11 ; 17)$ and $\mathrm{t}(5 ; 17)$ translocations, which result in NUMA/RAR $\alpha$ and NPM/RAR $\alpha$ fusion products, respectively, are associated with a poor response to ATRA (6) and unfavorable prognosis (7). In addition, Kurian et al (18) reported that an M3v case secondary to breast cancer, which carried $t(15 ; 17)$ (q13;12) and no PML/RAR $\alpha$ fusion transcripts, was resistant to ATRA. The present report described an M3v case with variant ins $(17 ; 15)(q 12 ; q 14 q 22)$ translocation, in which a segment of $15 q$, including the PML gene, was inserted into the RAR $\alpha$ gene on 17q. Among the reported APL cases with insertion, ins $(15 ; 17)$ is more common than ins $(17 ; 15)(19)$. To the best of our knowledge, only seven APL cases with ins $(17 ; 15)$ have been reported and all of these cases have been confirmed by FISH to carry insertions that can result in RAR $\alpha /$ PML fusion products. By conventional chromosomal analysis, four cases were found to have no abnormalities on chromosomes 15 and 17, and three cases had ins(17;15)(q21;q12q22), ins(17;15)(q21;q15q22) and ins $(17 ; 15)(q 21 ; q 14 q 22)(20,21)$, respectively. In the case with ins $(17 ; 15)(q 21 ; q 14 q 22)(19)$, the RAR $\alpha / P M L$ fusion transcripts were not detected by RT-PCR; therefore, there may be certain discrepancies between FISH and RT-PCR results, as found in the present case. The aforementioned and present cases exhibited complete remission following treatment with ATRA. The findings suggest that the rare variant ins $(17 ; 15)$ has no adverse effect on the therapeutic response to ATRA and is therefore associated with favorable prognosis. The underlying mechanisms remain unclear and further studies are required for their elucidation. Although RT-PCR has higher sensitivity in the diagnosis of acute leukemia and the monitoring of minimal residual disease than FISH, it is necessary to compare and combine the results of RT-PCR and FISH studies when dealing with cases with atypical characteristics.

In this case, there were three additional chromosomal abnormalities: add(3)(q29), -7 and trisomy 21. It has been reported that trisomy 8 and 21 and abn(17) are the three most frequently observed aberrations, in addition to $t(15 ; 17)$, in APL (22-25). To date, there has only been one case report describing 3q29 involved in translocation changes, rather than additional changes (26). Monosomy 7(-7) has been identified in $40 \%$ of pediatric patients with myelodysplastic syndrome, while only $4-5 \%$ of the AML cases carry -7 or $\operatorname{del}(7 q)$, which, according to the literature, suggests a poor prognosis (27). In pediatric patients, trisomy 21 is predominantly found in AML in association with Down's syndrome. Only a few reported cases were not associated with Down's syndrome, and the majority of these cases were associated with a poor outcome (24). In the present case, the patient did not have Down's syndrome. Spell et al (28) reported an $\mathrm{M} 3 \mathrm{v}$ case with trisomy 21 as an additional change but the prognostic implication of trisomy 21 was not clearly illustrated. The effect of the presence of additional aberrations in APL on prognosis is still controversial $(18,27)$; however, the findings of the present study suggest that the additional cytogenetic abnormalities appear to have no adverse effect on the response to treatment with ATRA and the prognosis.

\section{References}

1. Diverio D, Lo Coco F, D'Adamo F, et al: Identification of DNA rearrangements at the retinoic acid receptor-alpha (RAR-alpha) locus in all patients with acute promyelocytic leukemia (APL) and mapping of APL breakpoints within the RAR-alpha second intron. Italian Cooperative Study Group 'GIMEMA'. Blood 79: 3331-3336, 1992

2. Yamanouchi J, Hato T, Niiya T, et al: A new four-way variant $\mathrm{t}(5 ; 17 ; 15 ; 20)(\mathrm{q} 33 ; \mathrm{q} 12 ; \mathrm{q} 22 ; \mathrm{q} 11.2)$ in acute promyelocytic leukemia. Int J Hematol 94: 395-398, 2011. 
3. Rowley JD, Golomb HM and Dougherty C: $15 / 17$ translocation, a consistent chromosomal change in acute promyelocytic leukaemia. Lancet 1: 549-550, 1977.

4. de Thé H, Chomienne C, Lanotte M, Degos L and Dejean A: The $t(15 ; 17)$ translocation of acute promyelocytic leukaemia fuses the retinoic acid receptor alpha gene to a novel transcribed locus. Nature 347: 558-561, 1990.

5. Campbell LJ, Oei P, Brookwell R, et al: FISH detection of PML-RARA fusion in ins $(15 ; 17)$ acute promyelocytic leukemia depends on probe size. Biomed Res Int 2013: 164501, 2013

6. Rizzatti EG, Portieres FL, Martins SL, Rego EM, Zago MA and Falcão RP: Microgranular and t(11;17)/PLZF-RARalpha variants of acute promyelocytic leukemia also present the flow cytometric pattern of CD13, CD34 and CD15 expression characteristic of PML-RARalpha gene rearrangement. Am J Hematol 76: 44-51, 2004

7. Redner RL: Variations on a theme: The alternate translocations in APL. Leukemia 16: 1927-1932, 2002.

8. Zheng L, Xue Y,Li J, et al: Application of metaphase-fluorescence in situ hybridization to the diagnosis of acute promyelocytic leukemia and the detection of minimal residual disease. Zhongguo Shi Yan Xue Ye Xue Za Zhi 8: 180-184, 2000 (In Chinese).

9. Gallagher RE, Li YP, Rao S, et al: Characterization of acute promyelocytic leukemia cases with PML-RAR alpha break/fusion sites in PML exon 6: Identification of a subgroup with decreased in vitro responsiveness to all-trans retinoic acid. Blood 86: 1540-1547, 1995.

10. Avvisati G, Lo Coco F and Mandelli F: Acute promyelocytic leukemia: Clinical and morphologic features and prognostic factors. Semin Hematol 38: 4-12, 2001.

11. Guglielmi C, Martelli MP, Diverio D, et al: Immunophenotype of adult and childhood acute promyelocytic leukaemia: Correlation with morphology, type of PML gene breakpoint and clinical outcome. A cooperative Italian study on 196 cases. B J Haematol 102: 1035-1041, 1998.

12. Tallman MS, Kim HT, Montesinos P, et al: Does microgranular variant morphology of acute promyelocytic leukemia independently predict a less favorable outcome compared with classical M3 APL? A joint study of the North American Intergroup and the PETHEMA Group. Blood 116: 5650-5659, 2010.

13. Yoshii M, Ishida M, Yoshida T, et al: Clinicopathological features of acute promyelocytic leukemia: An experience in one institute emphasizing the morphological and immunophenotypic changes at the time of relapse. Int J Clin Exp Pathol 6: 2192-2198, 2013.

14. Invernizzi R, Iannone AM, Bernuzzi S, et al: Acute promyelocytic leukemia: Morphological and clinical features. Haematologica 78: 156-161, 1993.

15. Fang J, Chen SJ, Tong JH, Wang ZG, Chen GQ and Chen Z: Treatment of acute promyelocytic leukemia with ATRA and $\mathrm{As}_{2} \mathrm{O}_{3}$ : A model of molecular target-based cancer therapy. Cancer Biol Ther 1: 614-620, 2002.

16. Kim MJ, Cho SY, Lim G, et al: A rare case of microgranular acute promyelocytic leukemia associated with ider(17)(q10) $\mathrm{t}(15 ; 17)$ in an old-age patient. Korean J Lab Med 31: 86-90, 2011.
17. Tang H, Liu ZF and Pan JL: The clinical and experimental research of 2 cases of acute promyelocytic leukemia with atypical t(15;17) translocation. Guo Ji Shu Xue Ji Xue Ye Xue Za Zhi 35: 315-317, 2012 (In Chinese).

18. Kurian S, Hogan TF, Bleigh OC, Dowdy YG, Merghoub T, Pandolfi PP and Wenger SL: Atypical t(15;17)(q13;q12) in a patient with all-trans retinoic acid refractory secondary acute promyelocytic leukemia: A case report and review of the literature. Cancer Genet Cytogenet 138: 143-148, 2002.

19. Chen SN, Xue YQ, Wu YF and Pan JL: Cytogenetic and molecular genetic studies on a variant of $\mathrm{t}(15 ; 17)$, ins $(17 ; 15)(\mathrm{q} 21 ; \mathrm{q} 14 \mathrm{q} 22)$, in an acute promyelocytic leukemia patient. Zhonghua Yi Xue Yi Chuan Xue Za Zhi 21: 77-79, 2004 (In Chinese).

20. Grimwade D, Gorman P, Duprez E, et al: Characterization of cryptic rearrangements and variant translocations in acute promyelocytic leukemia. Blood 90: 4876-4885, 1997.

21. Grimwade D, Biondi A, Mozziconacci MJ, et al: Characterization of acute promyelocytic leukemia cases lacking the classic $\mathrm{t}(15 ; 17)$ : Results of the European Working Party. Groupe Français de Cytogénétique Hématologique, Groupe de Français d'Hematologie Cellulaire, UK Cancer Cytogenetics Group and BIOMED 1 European Community-Concerted Action 'Molecular Cytogenetic Diagnosis in Haematological Malignancies'. Blood 96: 1297-1308, 2000

22. Haimi M, Elhasid R, Weyl Ben-Arush M, Brill-Zamir R, Laevski I and Gershoni-Baruch R: Derivative (7)t(7;8)(q34;q21): An additional chromosome aberration in acute promyelocytic leukemia-prognostic influence debated. Cancer Genet Cytogenet 153: 81-83, 2004.

23. Cervera J, Montesinos P, Hernández-Rivas JM, et al: Additional chromosome abnormalities in patients with acute promyelocytic leukemia treated with all-trans retinoic acid and chemotherapy. Haematologica 95: 424-431, 2010.

24. Wan TS, Ma SK, Au WY, Liu HS, Chan JC and Chan LC: Trisomy 21 and other chromosomal abnormalities in acute promyelocytic leukemia. Cancer Genet Cytogenet 140: 170-173, 2003.

25. Bastos EF, Silva LA, Ramos MC, et al: Trisomy 11 as an additional chromosome alteration in a child with acute promyelocytic leukemia with poor prognosis. Case Rep Genet 2012: 659016, 2012

26. Xue Y, Lu D, Yuan YZ, Guo Y and Xie X: A rare variant translocation $\mathrm{t}(3 ; 8)$ (q29;q22) without AML1/ETO fusion transcript in a case of oligoblastic leukemia. Leuk Res 22: 1015-1019, 1998.

27. Hasle H, Alonzo TA, Auvrignon A, et al: Monosomy 7 and deletion $7 \mathrm{q}$ in children and adolescents with acute myeloid leukemia: An international retrospective study. Blood 109: 4641-4647, 2007

28. Spell DW, Velagaleti GV, Jones DV and Velasquez WS: Translocation $(15 ; 17)$ and trisomy 21 in the microgranular variant of acute promyelocytic leukemia. Cancer Genet Cytogenet 132: 74-76, 2002. 